\title{
miR-155 Is Associated with the Leukemogenic Potential of the Class IV Granulocyte Colony-Stimulating Factor Receptor in CD34+ Progenitor Cells
}

\author{
HaiJiao Zhang, ${ }^{1}$ Lilia Goudeva, ${ }^{1}$ Stephan Immenschuh, ${ }^{1}$ Axel Schambach, ${ }^{2,3}$ Julia Skokowa, ${ }^{4}$ \\ Britta Eiz-Vesper, ${ }^{1}$ Rainer Blasczyk, ${ }^{1,3}$ and Constança Figueiredo ${ }^{1,3}$ \\ ${ }^{1}$ Institute for Transfusion Medicine, Hannover Medical School, Hannover, Germany; ${ }^{2}$ Institute of Experimental Hematology, \\ Hannover Medical School, Hannover, Germany; ${ }^{3}$ Excellence Cluster "From Regenerative Biology to Reconstructive Therapies," \\ REBIRTH, Hannover Medical School, Hannover, Germany; and ${ }^{4}$ Department of Oncology, Hematology, Immunology, Rheumatology \\ and Pulmonology, University Hospital of Tübingen, Tübingen, Germany
}

\begin{abstract}
Granulocyte colony-stimulating factor (G-CSF) is a major regulator of granulopoiesis on engagement with the G-CSF receptor (G-CSFR). The truncated, alternatively spliced, class IV G-CSFR (G-CSFRIV) has been associated with defective differentiation and relapse risk in pediatric acute myeloid leukemia (AML) patients. However, the detailed biological properties of G-CSFRIV in human $\mathrm{CD}_{4}{ }^{+}$hematopoietic stem and progenitor cells (HSPCs) and the potential leukemogenic mechanism of this receptor remain poorly understood. In the present study, we observed that G-CSFRIV-overexpressing (G-CSFRIV') HSPCs demonstrated an enhanced proliferative and survival capacity on G-CSF stimulation. Cell cycle analyses showed a higher frequency of G-CSFRIV ${ }^{+}$

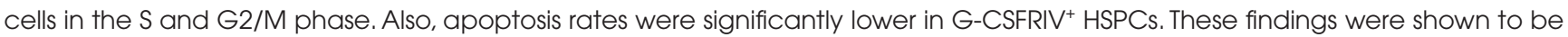
associated with a sustained Stat5 activation and elevated miR-155 expression. In addition, G-CSF showed to further induce G-CSFRIV and miR-155 expression of peripheral blood mononuclear cells isolated from AML patients. A Stat5 pharmacological inhibitor or ribonucleic acid (RNA) interference-mediated silencing of the expression of miR-155 abrogated the aberrant proliferative capacity of the G-CSFRIV ${ }^{+}$HSPCs. Hence, the dysregulation of Stat5/miR-155 pathway in the G-CSFRIV ${ }^{+}$HSPCs supports their leukemogenic potential. Specific miRNA silencing or the inhibition of Stat5-associated pathways might contribute to preventing the risk of leukemogenesis in G-CSFRIV ${ }^{+}$HSPCs. This study may promote the development of a personalized effective antileukemia therapy, in particular for the patients exhibiting higher expression levels of G-CSFRIV, and further highlights the necessity of prescreening the patients for G-CSFR isoforms expression patterns before G-CSF administration.
\end{abstract}

Online address: http://www.molmed.org

doi: $10.2119 / \mathrm{molmed} .2014 .00146$

\section{INTRODUCTION}

Granulocyte colony-stimulating factor (G-CSF) plays an important role in the homeostasis of granulopoiesis in the steady state and during emergencies (1). G-CSF supports the production, survival, proliferation, differentiation and mobilization of myeloid progenitor and precursor cells via the G-CSF receptor (G-CSFR). Clinically, G-CSF is commonly used to treat severe congenital neutropenia and to mobilize hematopoietic stem and progenitor cells (HSPCs) for transplantation. Recently, G-CSF was also used to facilitate hematopoietic recovery after transplantation, as a

Address correspondence to Constança Figueiredo, Institute for Transfusion Medicine, CarlNeuberg-Str. 1, D-30625 Hannover, Germany. Phone: +49-51 1-532-971 1; Fax: +49-51 1-532-

9701; E-mail: figueiredo.constanca@mh-hannover.de.

Submitted July 22, 2014; Accepted for publication December 15, 2014; Epub (www.molmed.org) ahead of print December 16, 2014.

\section{The Feinstein Institute for Medical Research} Empowering Imagination. Pioneering Discovery.

chemosensitizer to prime leukemia cells and as a primary prophylactic treatment to prevent chemotherapy-related neutropenia in patients with a high risk of febrile neutropenia (2). However, the potential leukemogenic role of G-CSFR variants has now become a major concern (3).

The wild-type G-CSFR (also known as class I G-CSFR [G-CSFRI]) consists of an extracellular domain, a transmembrane domain and a cytoplasmic domain. The C-terminal end of the G-CSFRI cytoplasmic domain contains four conserved tyrosine (Y) residues (Y704, Y729, Y744 and Y764), which form potential binding sites for signaling molecules (4). Furthermore, the dileucine motif at residues $749-754$ facilitates the internal- 
ization of the receptor (5). In response to G-CSF, the G-CSFR forms homodimers and leads to rapid Jak and Lyn phosphorylation and the activation of the signal transducers and activators of transcription (Stats)/suppressor of cytokine signaling (SOCS), mitogenactivated protein kinase/extracellular signal-regulated kinase (ERK) and phosphatidylinositol 3-kinase / AKT cascades $(2,6)$.

To date, seven alternatively spliced G-CSFR messager RNA (mRNA) isoforms have been identified in humans, but only G-CSFRI and the class IV G-CSFR (G-CSFRIV) were detectable in hematopoietic cells (7). Human myeloid cells express mainly G-CSFRI, and the expression of this receptor increases during normal granulogenesis (8). However, the blast cells from acute myeloid leukemia (AML) and myelodysplastic syndrome patients as well as leukemic cell lines (HL60, NB4 and EM3) had elevated G-CSFRIV:G-CSFRI mRNA ratios compared with normal immature myeloid cells $(8,9)$. Notably, a role of G-CSFRIV in human myeloid leukemia has been recently suggested from the following two studies: (a) the overexpression of G-CSFRIV favors the expansion of monosomy 7 clones in response to G-CSF (10), and (b) pediatric AML patients with G-CSFRIV overexpression have a higher incidence of relapse with G-CSF administration (11). Therefore, there is a great need to evaluate the biological properties and leukemogenic potential of G-CSFRIV in primary $\mathrm{CD} 34^{+} \mathrm{HSPCs}$.

MicroRNAs (miRNAs) are short (20-25 nt) noncoding RNAs that posttranscriptionally modulate the expression of multiple target genes. Emerging evidence shows that specific miRNAs are involved in hematopoiesis under physiological and pathological conditions (12). Among the miRNAs expressed in hematopoietic cells, miR-155 is one of the most abundant and has been linked to hematopoietic lineage differentiation and hematopoietic malignancy (13). Therefore, in the present study, we evaluated whether the expression pattern of miR-155 is associated with the leukemogenic potential of G-CSFRIV. Furthermore, we investigated the feasibility of reversing the G-CSFRIV effect with a Stat5 inhibitor and RNA interference technology targeting miR-155.

\section{MATERIALS AND METHODS}

\section{Cell Isolation and Culture}

Peripheral blood CD $34^{+}$HSPCs from 40 healthy human leukocyte antigen (HLA)-typed donors and peripheral blood mononuclear cells (PBMCs) from 13 AML patients and 8 healthy donors were collected after obtaining informed consent and the approval of the local ethics committee of the Hannover Medical School. The CD $34^{+}$HSPCs were isolated with a human CD34 microbead kit (Miltenyi Biotec; Bergisch Gladbach, Germany). The isolated CD $34^{+}$HSPCs were cultured in HSPC culture medium consisting of RPMI-1640 (BioWhittaker/ Cambrex, Hess Oldendorf, Germany) supplemented with 5\% human AB serum (C.C. Pro, Neustadt, Germany), thrombopoietin (100 ng/mL), Fms-like tyrosine kinase 3 ligand (100 ng/mL), stem cell factor (100 $\mathrm{ng} / \mathrm{mL}$ ) and interleukin (IL)-6 (50 $\mathrm{ng} / \mathrm{mL}$ ) for up to $2 \mathrm{~d}$ to maintain an undifferentiated state of the cells. The AML cases were categorized according to World Health Organization tumor classification (14) by using anonymous clinical reports. The PBMCs were cultured in RPMI-1640 supplemented with $5 \%$ human $\mathrm{AB}$ serum in the presence or absence of G-CSF. All of the recombinant cytokines were purchased from PeproTech (Rocky Hill, NJ, USA).

\section{Lentiviral Vector Production and Transduction}

A lentiviral vector was used for the stable expression of G-CSFRI or G-CSFRIV. Briefly, G-CSFRI and G-CSFRIV sequences were cloned into the lentiviral vector (pRRLSIN.cPPT.PGK-GFP.WPRE) containing enhanced green fluorescent protein $(G F P)$ as a reporter gene. Lentiviral particles were produced by transfecting $5 \times 10^{6}$ human embryonic kidney (HEK) 293T cells with $10 \mu \mathrm{g}$ G-CSFRI- or
G-CSFRIV-encoding vector, $9 \mu \mathrm{g}$ psPAX2 gag/pol plasmid, and $3 \mu \mathrm{g}$ pMD2G encoding the VSV-G envelope protein. A short-hairpin RNA (shRNA) targeting miR-155 (shmiR-155) was cloned into the pRRL.PPT.SF.DsRedEx.pre vector containing enhanced DsRed fluorescent protein as a reporter gene. The shRNA-expressing vector was produced by HEK 293T cells after cotransfection with $3 \mu \mathrm{g}$ pMD2G, $5 \mu \mathrm{g}$ pRSV-Rev and $9 \mu \mathrm{g}$ pMDLg/pRRE. The psPAX2, pMD2G, pRSV-Rev and pMDLg/pRRE plasmids were purchased from Addgene (Cambridge, MA, USA).

After 1 and $2 \mathrm{~d}$, the viral vector-containing supernatants were collected, filtered and concentrated by ultracentrifugation (Optima L-100 XP; Beckman Coulter, Krefeld, Germany) at 30,000 $g$ for $4 \mathrm{~h}$ at $4^{\circ} \mathrm{C}$.

\section{Lentiviral Vector Transduction}

A total of $1-2 \times 10^{6}$ cells/well were seeded into a RetroNectin-coated (Takara, Otsu, Japan) 12-well plates in the HSPC culture medium and infected with the lentiviral vector in the presence of $8 \mu \mathrm{g} / \mathrm{mL}$ protamine sulfate (Sigma-Aldrich, Steinheim, Germany). After $16 \mathrm{~h}$, the cells were washed, and fresh medium was added to the cells. Subsequently, the transduced CD34 ${ }^{+}$HSPCs expressing GFP were enriched via flow cytometry with fluorescence-activated cell sorting (FACS) (MoFlo or XDP sorter; Beckman Coulter).

\section{Flow Cytometry}

Cells were analyzed for the expression of specific surface antigens via FACS (FACSCanto II; BD Biosciences, San Jose, CA, USA). The following monoclonal antibodies were used: anti-CD11b, antiCD11c, anti-CD14, anti-CD33 and antiCD114 (all purchased from BioLegend, San Diego, CA, USA). The data were analyzed with the FACSDiva software 6.0 (BD Biosciences) or FlowJo 7.6 software (Tree Star, Ashland, OR, USA).

\section{Proliferation Assay}

Cells were labeled with $5 \mu \mathrm{mol} / \mathrm{L}$ cell proliferation dye (CPD) eFluor 670 (eBiosciences, San Diego, CA, USA) and cultured with different stimulations for $3 \mathrm{~d}$. 
The cells were then harvested and analyzed via FACS.

\section{Bromodeoxyuridine Incorporation Assay}

Cells $\left(1 \times 10^{5} /\right.$ well $)$ were cultured in a 48-well plate and stimulated with 10, 100 or $400 \mathrm{ng} / \mathrm{mL} \mathrm{G-CSF}$. After $1 \mathrm{~d}$, the cells were incubated with $10 \mu \mathrm{mol} / \mathrm{L}$ bromodeoxyuridine (BrdU) (BD Biosciences) for $3 \mathrm{~h}$ and then washed with phosphatebuffered saline and fixed with ice-cold $70 \%$ ethanol overnight at $-20^{\circ} \mathrm{C}$. The fixed cells were pelleted and denatured by incubating with $2 \mathrm{~mol} / \mathrm{L} \mathrm{HCL}$ for $20 \mathrm{~min}$, followed by neutralization with $0.1 \mathrm{~mol} / \mathrm{L}$ boric acid (Sigma-Aldrich) for $2 \mathrm{~min}$. The incorporated BrdU was detected with an allophycocyanin (APC)-conjugated antiBrdU antibody (BioLegend), and the cells were analyzed via FACS. An anti-CD114 antibody was used to distinguish the G-CSFR variant-overexpressing or G-CSFR nonexpressing cells.

\section{Cell Cycle Analysis}

Cells were harvested, fixed with precooled $70 \%$ ethanol at $4{ }^{\circ} \mathrm{C}$ overnight and then stained with $50 \mathrm{ng} / \mathrm{mL}$ propidium iodide (PI) (Sigma-Aldrich) and 100 ng/mL RNase (Sigma-Aldrich) or anti-Ki67 antibody (BioLegend) at $37^{\circ} \mathrm{C}$ for $30 \mathrm{~min}$ in the dark. The cell cycle distribution was evaluated with FACS and analyzed by using the FlowJo software.

\section{Apoptosis Assay}

The detection of apoptotic cells was performed by staining the cells with annexin V/PI. Cells were stained with APC-labeled annexin V and PI according to the manufacturer's instructions. The ratios of viable cells and apoptotic cells were determined by FACS analysis. Annexin V and PI were purchased from Biolegend.

\section{Colony-Forming Assay}

A total of 500 cells per well were seeded into a six-well plate with $1.1 \mathrm{~mL}$ Methocult H4035 methylcellulose medium (StemCell Technologies, Vancouver, Canada) and incubated in $5 \% \mathrm{CO}_{2}$ with high humidity at $37^{\circ} \mathrm{C}$. After 10 and $14 \mathrm{~d}$, colonies ( $>50$ cells) and total cells were counted, harvested, stained with cell surface markers and analyzed by FACS. Cytospin (Thermo Fisher Scientific, Waltham, MA, USA) cell slides were stained with May-Grünwald-Giemsa solution (Sigma-Aldrich) according to the protocol provided.

\section{Signal Transduction Assay}

Intracellular signaling pathways were evaluated using BD Phosflow technology (BD Biosciences). Briefly, cells were incubated in serum-free RPMI medium for $1 \mathrm{~h}$ and stimulated with G-CSF (100 ng/mL) alone or together with dimethyl sulfoxide (DMSO) (Sigma-Aldrich) or a Stat5 inhibitor $\left(100 \mu \mathrm{mol} / \mathrm{L}, N^{\prime}-[(4-\mathrm{Oxo}-4 \mathrm{H}-\right.$ chromen-3-yl) methylene] nicotinohydrazide; EMD Millipore, Billerica, CA, USA) for various time periods (0-150 $\mathrm{min}$ ) at $37^{\circ} \mathrm{C}$. The cells were then fixed with Cytofix buffer (BD Biosciences) for $10 \mathrm{~min}$, permeabilized with $90 \%$ methanol on ice for $30 \mathrm{~min}$, stained with PE-conjugated anti-phosphorylated Stat5 (anti-pStat5; pY694), PE-conjugated anti-pStat3 (pY705), APC-conjugated anti-pAKT and APC-conjugated anti-pERK1/2 antibodies in the presence of a phosphatase inhibitor (BD Biosciences) for $1 \mathrm{~h}$ at $4^{\circ} \mathrm{C}$ and analyzed by FACS. All of the antibodies were purchased from BD Biosciences.

\section{Evaluation of miRNA and mRNA Levels}

Total cellular RNA for both miRNA and mRNA analyses was isolated and purified with the mirVana ${ }^{\mathrm{TM}}$ miRNA Isolation Kit (Life Technologies [now Thermo Fisher Scientific]) according to the manufacturer's instructions.

To detect miR-155-5p expression, a TaqMan microRNA assay was performed as previously described (15). Briefly, total RNA was reverse-transcribed into complementary deoxyribonucleic acid (cDNA) by using a TaqMan microRNA Reverse Transcription Kit and a miRNAspecific primer, followed by real-time polymerase chain reaction (PCR) with TaqMan probes and a TaqMan Universal PCR Master Mix using a StepOnePlus real-time PCR system according to the manufacturer's instructions. For the mRNA quantification, total RNA was transcribed into cDNA with a highcapacity cDNA reverse-transcriptase kit. Real-time PCR was performed by using the reverse transcription-PCR master mix and a FAM (carboxyfluorescein)-labeled minor groove binding TaqMan probe. U6 or glyceraldehyde-3-phosphate dehydrogenase (GAPDH) levels were used as endogenous control of miR-155 or mRNA levels, respectively. The G-CSFRI and G-CSFRIV primers were purchased from TIB Molbiol (Berlin, Germany). The other materials were purchased from Life Technologies (now Thermo Fisher Scientific). Primers and probes are listed in Supplementary Table S1.

\section{Cytokine Secretion Assay}

Cells were cultured with G-CSF plus DMSO as a control or G-CSF plus the Stat 5 inhibitor for 1 and $3 \mathrm{~d}$. Supernatants of the cell culture were collected and analyzed for cytokine secretion by using Luminex technology (human cytokine 12plex panel; EMD Millipore) and a Luminex 200 instrument (Invitrogen/Life Technologies [Thermo Fisher Scientific]).

\section{Statistical Analysis}

All of the data were expressed as the mean \pm standard deviation (SD). Statistical analyses were performed using two-tailed Student $t$ tests run on the GraphPad Prism 5 software (GraphPad Software, San Diego, CA, USA). The levels of significance were expressed as $p$ values $\left({ }^{*} p<\right.$ $0.05,{ }^{* *} p<0.01$ and $\left.{ }^{* * *} p<0.001\right)$.

All supplementary materials are available online at www.molmed.org.

\section{RESULTS}

\section{G-CSFRIV Promotes HSPC Proliferation and Cell Cycle Progression}

In contrast to leukemic cells, healthy CD34 ${ }^{+}$HSPCs present very low G-CSFRIV: G-CSFRI gene expression ratios (16). An alteration in the G-CSFRIV:G-CSFRI ratio in HPSCs was mimicked in vitro by the overexpression of either the G-CSFRI or 
G-CSFRIV isoform in healthy CD34 HSPCs. To characterize the proliferative potential of the G-CSFRI- and G-CSFRIVoverexpressing (G-CSFRI ${ }^{+}$and G-CSFRIV ${ }^{+}$) HSPCs, cell counting, BrdU incorporation and labeling assays with CPD eFluor 670 were performed. Higher proliferation rates were observed in the G-CSFRIV ${ }^{+}$ HSPCs compared with the G-CSFRI ${ }^{+}$ HSPCs, as indicated by the increased cell counts (percentage of increase: $26.4 \%$, $p=0.0004$, at $100 \mathrm{ng} / \mathrm{mL}$ and $33.6 \%, p=$ 0.0497, at $400 \mathrm{ng} / \mathrm{mL}$ ) (Figure 1A), the enhanced BrdU incorporation (percentage of increase: $15.8 \%, p=0.0142$, at $100 \mathrm{ng} / \mathrm{mL}$ and $14.1 \%, p=0.0022$, at $400 \mathrm{ng} / \mathrm{mL}$ ) (Figure $1 \mathrm{~B}$ ) and the marked reduction of the CPD eFluor 670 mean fluorescence intensity (MFI) (percentage of change: $31.4 \%$, $p=0.0026$, at $100 \mathrm{ng} / \mathrm{mL}$ and $27.1 \%, p=$ 0.0274 , at $400 \mathrm{ng} / \mathrm{mL}$ ) (Figures 1C, D) on G-CSF stimulation. No significant alterations in the proliferation rates were observed when the HSPCs were cultured in the presence of IL-6 or among the G-CSFR nonexpressing cells and the nontransduced cells used as negative controls (Figures 1A, C, D and Supplementary Figure S1). The cell cycle analysis demonstrated an increase in the percentage of cells in the $S$ phase $\left(18.60 \pm 1.67 \% \mathrm{G}^{-C S F R I}{ }^{+}\right.$versus $24.53 \pm$ $2.85 \%$ G-CSFRIV $\left.{ }^{+}, p=0.0752\right)$ and the G2/M phase $\left(10.11 \pm 0.30 \% \mathrm{G}-\mathrm{CSFRI}^{+}\right.$ versus $12.27 \pm 0.57 \% \mathrm{G}_{-} \mathrm{CSFRIV}^{+}, p=$ $0.0451)$ and a decrease in the percentage of cells in G0/G1 phase $(67.04 \pm 1.21$ $\mathrm{G}-\mathrm{CSFRI}^{+}$versus $59.87 \pm 1.40 \mathrm{G}-\mathrm{CSFRIV}^{+}$, $p=0.0231)$ in the G-CSFRIV ${ }^{+}$HSPCs compared with the G-CSFRI ${ }^{+}$HSPCs (Figures $1 \mathrm{E}, \mathrm{F})$. Accordingly, a significant increase in Ki67 staining in the G-CSFRIV ${ }^{+}$ HSPCs was observed (Ki67 MFI: $169.0 \pm$ 47.44 G-CSFRI ${ }^{+}$versus $231.7 \pm 31.79$ $\mathrm{G}-C S F R I V^{+}, p=0.0316$ ) (Figures 1G, H). These data strongly suggest that G-CSFRIV promotes $\mathrm{CD} 34^{+}$HSPCs proliferation and cell cycle progression on G-CSF stimulation.

\section{G-CSFRIV Protects HSPCs from Apoptosis}

Truncated variants of the G-CSFR have been shown to confer resistance to apoptosis and contribute to leukemic trans-
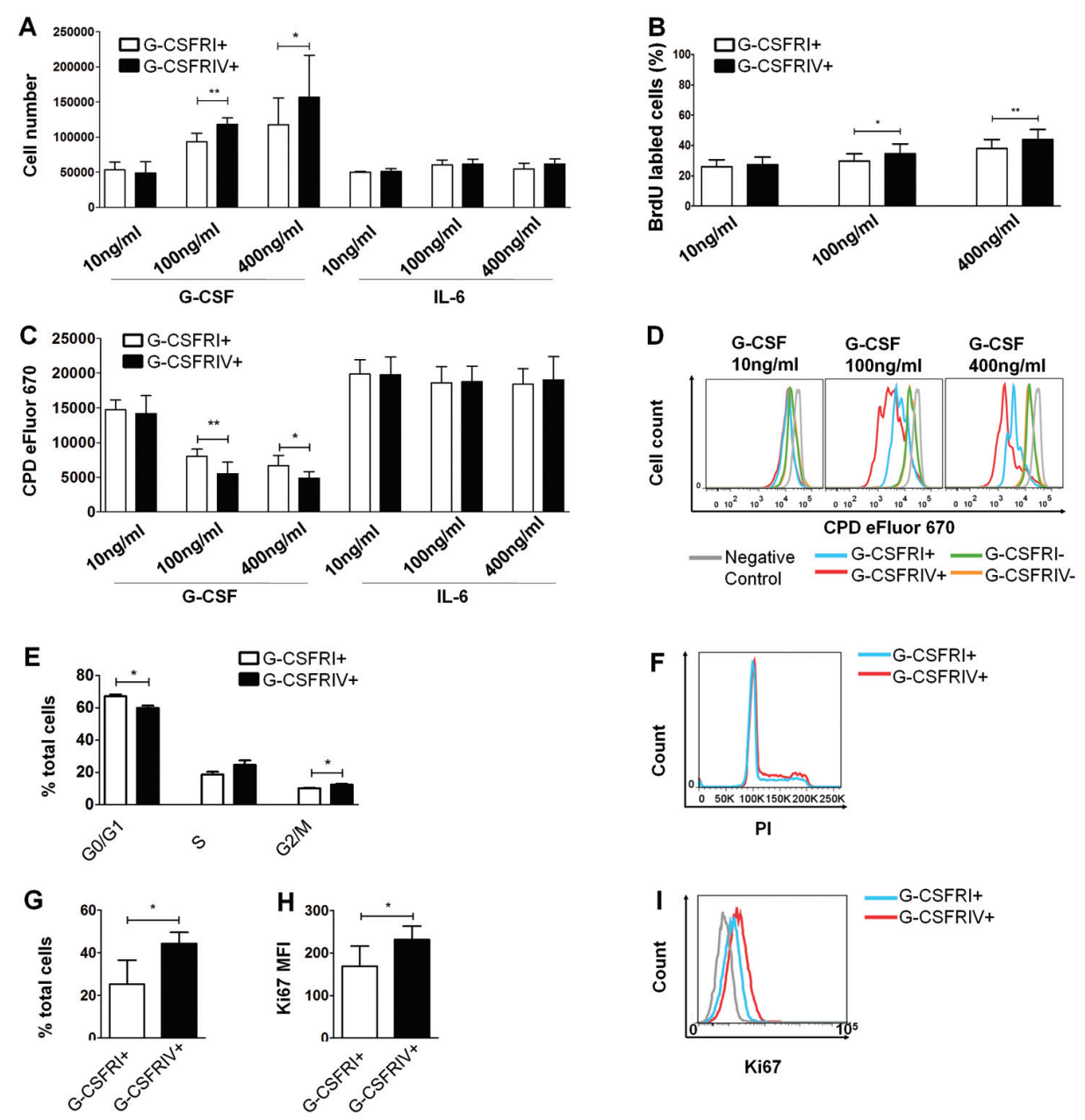

Figure 1. G-CSFRIV promotes HSPC proliferation and cell cycle progression. CD34 ${ }^{+} \mathrm{HSPCs}$ were transduced with G-CSFRI or G-CSFRIV encoding lentiviral vector. (A) Cells $\left(1 \times 10^{5}\right)$ were stimulated with 10, 100 and $400 \mathrm{ng} / \mathrm{mL}$ G-CSF or IL-6 for $3 \mathrm{~d}$. Graph displays total cell numbers counted under an optical microscope. (B) Cells $\left(1 \times 10^{5}\right)$ were stimulated with indicated G-CSF concentrations for $1 \mathrm{~d}$ and incorporated with BrdU for $3 \mathrm{~h}$ followed by antiBrdU antibody staining and FACS analysis. Anti-CD 114 antibody was used to distinguish G-CSFR overexpressing and G-CSFR nonexpressing cells. Graph displays percentages of BrdU-labeled cells in the total G-CSFRI ${ }^{+}$and G-CSFRIV ${ }^{+}$population. (C) Cells $\left(1 \times 10^{5}\right)$ were labeled with the CPD eFluor 670 and stimulated with G-CSF or IL-6 for $3 \mathrm{~d}$. Cell proliferation was measured by FACS. GFP expression was used to distinguish G-CSFR overexpressing and G-CSFR nontransduced cells. Graph displays MFI of CPD eFluor 670 on G-CSFRI ${ }^{+}$and G-CSFRIV ${ }^{+}$cells. (D) Representative histograms of CPD efluor 670 fluorescence levels detected on nonstimulated (negative control), G-CSFRI', G-CSFRIV ${ }^{+}$, G-CSFRI ${ }^{-}$and G-CSFRIV ${ }^{-}$ HSPCs. Graphs above display mean \pm SD of the data acquired in six independent experiments. (E) Enriched G-CSFRI ${ }^{+}$and G-CSFRIV ${ }^{+} \operatorname{HSPCs}\left(1.5 \times 10^{5}\right)$ were cultured with $100 \mathrm{ng} / \mathrm{mL}$ G-CSF for $3 \mathrm{~d}$ and analyzed for cell cycle distribution and Ki67 expression.

Graph displays percentages of cells in each phase of the cell cycle. (F) Representative histogram of the cell cycle distribution. (G) Percentages of Ki67-labeled cells of total G-CSFRI ${ }^{+}$ and G-CSFRIV ${ }^{+}$HSPC population. (H) Ki67 MFI of G-CSFRI ${ }^{+}$and G-CSFRIV ${ }^{+}$HSPCs. (I) Representative histogram of Ki67 expression on G-CSFRI ${ }^{+}$and G-CSFRIV ${ }^{+}$HSPCs. Data shown are mean \pm SD of three independent experiments. Statistically significant differences were calculated using a two-tailed Student $t$ test and are shown with asterisks $\left({ }^{*} p<0.05\right.$ and ${ }^{* *} p<$ 0.01). G-CSFRI', G-CSFRI nontransduced; G-CSFRIV', G-CSFRIV nontransduced. 
formation (17). In this study, we investigated whether G-CSFRIV prevents apoptosis in HSPCs. Compared to the GCSFRI $^{+}$HSPCs, the G-CSFRIV ${ }^{+}$HSPC populations cultured with G-CSF had significantly higher rates of viable cells $\left(84.47 \pm 2.35 \% \mathrm{G}^{-C} \mathrm{SFRI}{ }^{+}\right.$versus $90.5 \pm$ $2.69 \%$ G-CSFRIV $\left.{ }^{+}, p=0.0324\right)$ and lower levels of apoptotic cells $(6.14 \pm 1.59 \%$ $\mathrm{G}-C S F R I^{+}$versus $3.48 \pm 1.12 \%$ G-CSFRIV ${ }^{+}$, $p=0.0378$, for early apoptosis and $9.32 \pm$ $0.76 \% \mathrm{G}-\mathrm{CSFRI}^{+}$versus $5.79 \pm 1.52 \%$ $\mathrm{G}-\mathrm{CSFRIV}^{+}, p=0.0482$, for later apoptosis) (Figures 2A-C). No differences in cell viability or apoptosis rates were detected between the G-CSFRI ${ }^{+}$and G-CSFRIV ${ }^{+}$ HSPCs cultured in the absence of G-CSF (Figures 2D-F). These data indicate that G-CSFRIV confers a survival advantage to HSPCs in a G-CSF-dependent manner.

\section{G-CSFRIV ${ }^{+}$HSPCs Demonstrate an Increased Colony-Forming Capacity and Impaired Myeloid Differentiation}

G-CSF-mediated cell proliferation and maturation have been shown to be uncoupled processes in murine cell lines that overexpressed G-CSFRIV (18). In concordance with the previous results, the G-CSFRIV ${ }^{+}$HSPCs cultured in myeloid lineage differentiation medium generated a significantly greater number of colonies and cells than the G-CSFRI ${ }^{+}$ HSPCs $\left(52 \pm 20 \mathrm{G}-\mathrm{CSFRI}^{+}\right.$versus $102 \pm 7$ $\mathrm{G}^{-C_{S F R I V}}, p=0.0087$, for colony number and 192,225 $\pm 140,768{\mathrm{G}-\mathrm{CSFRI}^{+}}$ver-

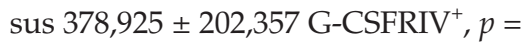
0.0084, for cell number) (Figures 3A, B). Furthermore, the G-CSFRIV ${ }^{+}$HSPCs exhibited lower expression levels of markers characteristic of myeloid maturation, such as CD11b, CD11c and CD14, and higher expression levels of progenitor cell markers, such as CD33 (Figures 3C-G). These data suggest that G-CSFRIV supports cell proliferation and is associated with delayed myeloid maturation.

\section{G-CSFRIV Mediates Aberrant Activation of the Stats, ERK and AKT Signaling Pathways}

Differential activation of signaling pathways between G-CSFRI and truncated

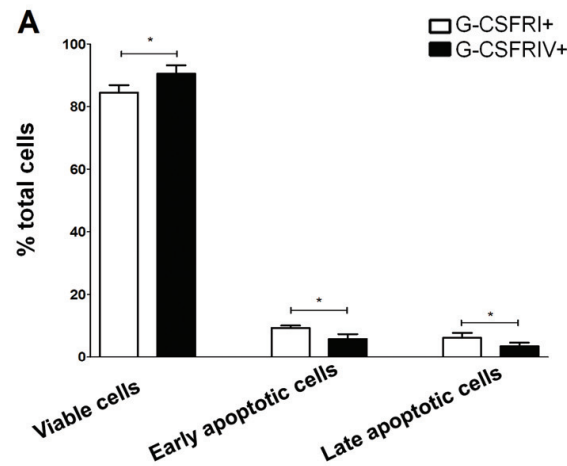

B

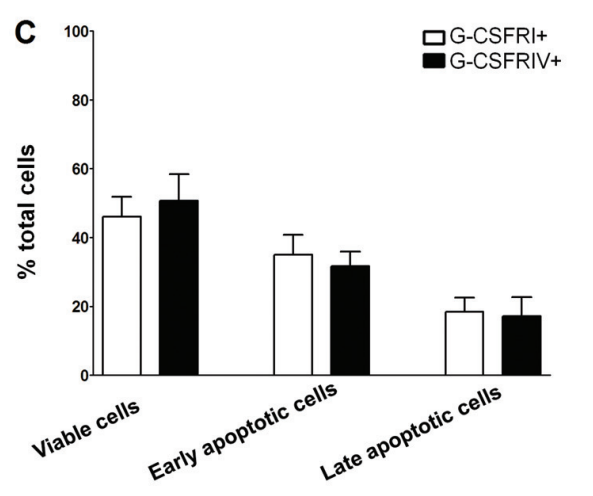

D

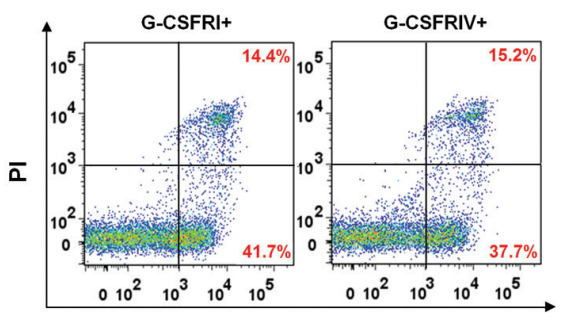

Annexin V

Figure 2. G-CSFRIV protects HSPCs from apoptosis. (A) Enriched G-CSFRI ${ }^{+}$and G-CSFRIV ${ }^{+}$ HSPCs $\left(1.5 \times 10^{5}\right)$ were cultured with $100 \mathrm{ng} / \mathrm{mL}$ G-CSF for $3 \mathrm{~d}$, stained with annexin V/PI and analyzed by FACS. Graph displays higher percentages of viable cells (annexin $\mathrm{V}^{-} / \mathrm{Pl}^{-}$) and lower percentages of early and late apoptotic (annexin $\mathrm{V}^{+} / \mathrm{Pl}^{-}$and annexin $\mathrm{V}^{+} / \mathrm{Pl}^{+}$) cells in the G-CSFRIV ${ }^{+}$HSPCs compared with G-CSFRI ${ }^{+}$HSPCs. (B) Representative dot plot of apoptosis analyses. On G-CSF stimulation, G-CSFRIV+ HSPCs showed decreased apoptotic rates in comparison to G-CSFRI HSPCs. (Viable cells: left lower quadrant; early apoptotic cells: right lower quadrant; and late apoptotic cells: right upper quadrant.) (C) G-CSFRl' and G-CSFRIV+ ${ }^{+}$SSPCs $\left(1.5 \times 10^{5}\right)$ were serum and cytokine starved for $1 \mathrm{~d}$ and analyzed for apoptotic activity. Graph displays similar percentages of viable, early apoptotic and late apoptotic cells in G-CSFRI ${ }^{+}$ and G-CSFRIV+ HSPCs. (D) Representative dot plot of annexin V/PI analyses showing comparable apoptotic activity of G-CSFRI ${ }^{+}$and G-CSFRIV ${ }^{+}$HSPCs without G-CSF stimulation. Data shown are mean \pm SD of three independent experiments. Statistically significant differences were calculated using a two-tailed Student $t$ test and are shown with asterisks ( $\left.{ }^{*} p<0.05\right)$.

variants of the G-CSFR has been previously reported (19). To understand the mechanisms contributing to the enhanced growth and survival of G-CSFRIV ${ }^{+}$ HSPCs, we investigated the phosphorylation of Stat3, Stat5, ERK1/2 and AKT, which are known to be important for G-CSF-induced hematopoiesis (20). Upon G-CSF stimulation, the G-CSFRIV ${ }^{+}$HSPCs demonstrated significantly attenuated levels of Stat3 phosphorylation compared with the G-CSFRI ${ }^{+}$HSPCs (for example, $131.9 \pm 49.79 \mathrm{G}-\mathrm{CSFRI}^{+}$versus $86.74 \pm$ 27.79 G-CSFRIV ${ }^{+}, p=0.0394$, at $20 \mathrm{~min}$ ) (Figure 4A). Notably, upon G-CSF stimula- tion, a sustained activation of Stat5 was observed in the G-CSFRIV ${ }^{+}$HSPCs compared with the G-CSFRI ${ }^{+}$HSPCs (for example, $70.83 \pm 15.29 \mathrm{G}-\mathrm{CSFRI}^{+}$versus $125.6 \pm 23.32 \mathrm{G}^{-C} \mathrm{SFRIV}{ }^{+}, p=0.0048$, at $90 \mathrm{~min}$ ) (Figure 4B). ERK1/2 activation was significantly delayed and lower in the G-CSFRIV ${ }^{+}$HSPCs compared with the G-CSFRI ${ }^{+}$HSPCs during the first $30 \mathrm{~min}$, but ERK1/2 activation then remained constant until later time points (150 $\mathrm{min}$ ) (Figure $4 \mathrm{C}$ ). The activation of AKT in G-CSFRI ${ }^{+}$ cells was rapid but transient, whereas no obvious AKT activation could be detected in the G-CSFRIV ${ }^{+}$cells (Figure 4D). 
A
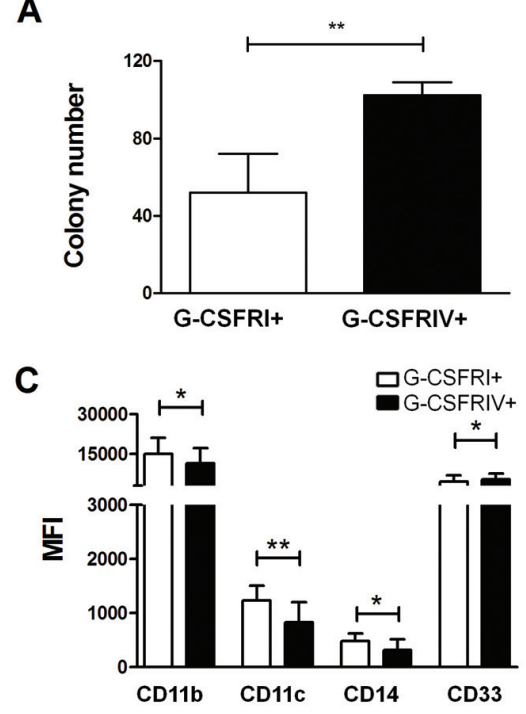

E

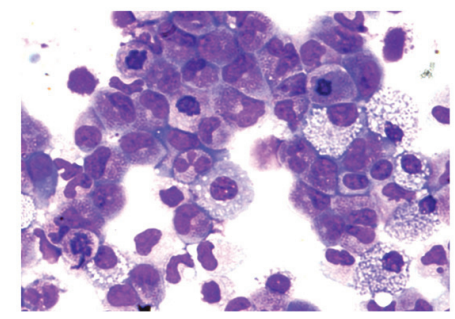

B

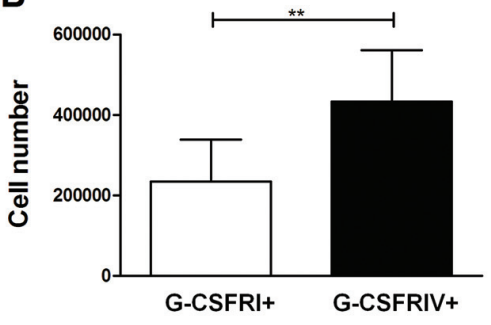

D

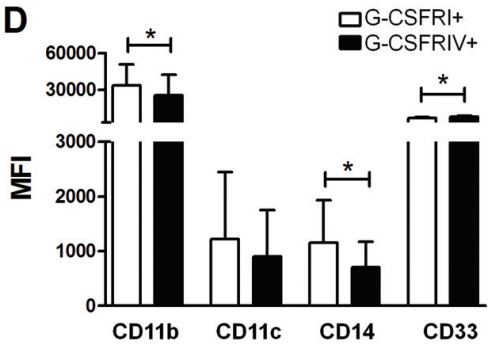

$\mathbf{F}$

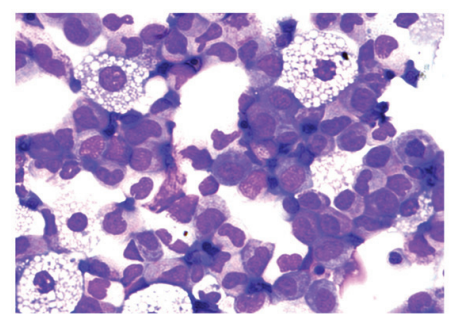

G

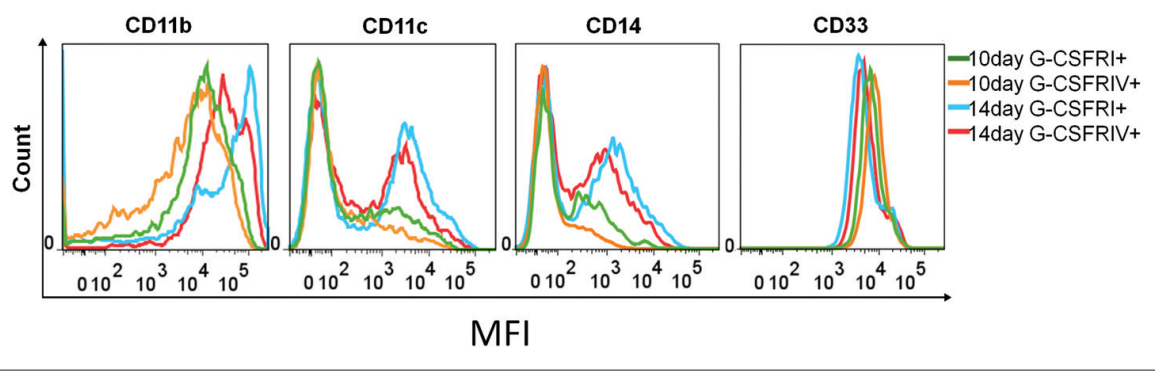

Figure 3. G-CSFRIV ${ }^{+}$HSPCs demonstrate an increased colony-forming capacity and im-

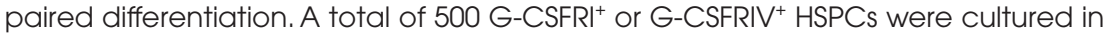
myeloid lineage differentiation medium for 10 or $14 \mathrm{~d}$. Graphs display colony numbers (A) and cell numbers (B) of G-CSFRI ${ }^{+}$and G-CSFRIV ${ }^{+}$HSPCs after 10 d. Graphs display MFI of CD11b, CD11c, CD14 and CD33 of G-CSFRI ${ }^{+}$and $G-C S F R I V^{+}$cells after $10 \mathrm{~d}(\mathrm{C})$ and $14 \mathrm{~d}$ (D). May-Grünwald-Giemsa staining of $G-C S F R l^{+}(E)$ and $G-C S F R I V+(F)$ cells after $10 \mathrm{~d}$ is shown. (J) Representative histograms for the expression of CD11b, CD1 1C, CD14 and CD33 of G-CSFRI ${ }^{+}$and G-CSFRIV ${ }^{+}$cells after 10 and $14 \mathrm{~d}$. Data are mean \pm SD of six independent experiments. Statistically significant differences were calculated by using a twotailed Student $t$ test and are shown with asterisks $\left({ }^{*} p<0.05\right.$ and $\left.{ }^{* *} p<0.01\right)$.

\section{Sustained Stat5 Activation in the G-CSFRIV ${ }^{+}$HSPCs Induces miR-155 Expression}

miR-155 is highly expressed in

hematopoietic progenitor cells and has been implicated in several hematological malignancies $(13,21)$. We therefore investi-

gated the levels of miR-155 in the $\mathrm{G}^{-C_{S F R I}}{ }^{+}$and G-CSFRIV ${ }^{+}$HSPCs to determine the expression pattern of miR-155 in relation to the growth and survival advantage of the G-CSFRIV ${ }^{+}$HSPCs. We observed increased relative quantification (RQ) values of miR-155 in the G-CSFRIV ${ }^{+}$
HSPCs in the presence of G-CSF (1.08 \pm $0.33 \mathrm{G}-C S F R I V^{+}$without G-CSF versus $1.41 \pm 0.36 \mathrm{G}^{-C S F R I V}{ }^{+}$with G-CSF, $p=$ 0.0031), whereas the G-CSFRI ${ }^{+}$HSPCs showed reduced RQ values of miR-155 (1.00 G-CSFRI ${ }^{+}$without G-CSF versus $0.70 \pm 0.11 \mathrm{G}-\mathrm{CSFRI}^{+}$with G-CSF, $p=$ 0.0037) (Figure 5A). Furthermore, the transcript levels of miR-155 candidate target genes, including TP53INP1, PU.1 and GFI-1, were measured. Accordingly, significantly reduced RQ values of TP53INP1

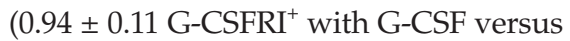
$0.64 \pm 0.04 \mathrm{G}^{-C S F R I V}{ }^{+}$with G-CSF, $p=$ 0.0420), PU.1 (1.08 $\pm 0.25 \mathrm{G}-\mathrm{CSFRI}^{+}$with

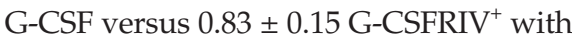
G-CSF, $p=0.0489)$ and GFI-1 (1.14 \pm 0.28 $\mathrm{G}^{-\mathrm{CSFRI}^{+}}$with $\mathrm{G}-\mathrm{CSF}$ versus $0.93 \pm 0.18$ $\mathrm{G}-C S F R I V^{+}$with G-CSF, $\left.p=0.0095\right)$ were observed in the G-CSFRIV ${ }^{+}$HSPC compared with the G-CSFRI ${ }^{+}$HSPCs on G-CSF stimulation (Figures 5B-D).

Notably, G-CSF failed to upregulate miR155 and inhibit miR-155 target genes in the presence of a Stat 5 inhibitor, which could inhibit Stat5 activation (Figures 5E-H and Supplementary Figure S2). These results indicate that the activation of Stat 5 is required for the induction of miR-155 in the G-CSFRIV $^{+}$HSPCs.

In addition, we compared the cytokine secretion profiles of the G-CSFRIV ${ }^{+}$and G-CSFRI ${ }^{+}$HSPCs. A significant increase in the level of CCL2 was observed in the G-CSFRIV $^{+}$HSPCs, which correlated with the activation of Stat5 (Table 1).

\section{Pharmacological or RNA}

Interference-Mediated Abrogation of the G-CSFRIV-Mediated

\section{Hyperproliferation of HSPCs}

An increased G-CSFRIV:G-CSFRI expression ratio in HSPCs may constitute a risk for leukemic transformation (11). The upregulation of miR-155 observed in the G-CSFRIV ${ }^{+}$HSPCs was dependent on Stat5 activation. Therefore, we evaluated the feasibility of inhibiting Stat5 activation or miR-155 expression to abrogate the increased cell proliferation demonstrated by the G-CSFRIV ${ }^{+}$HSPCs. As shown in Figure 6A, proliferation was significantly decreased in the presence of 

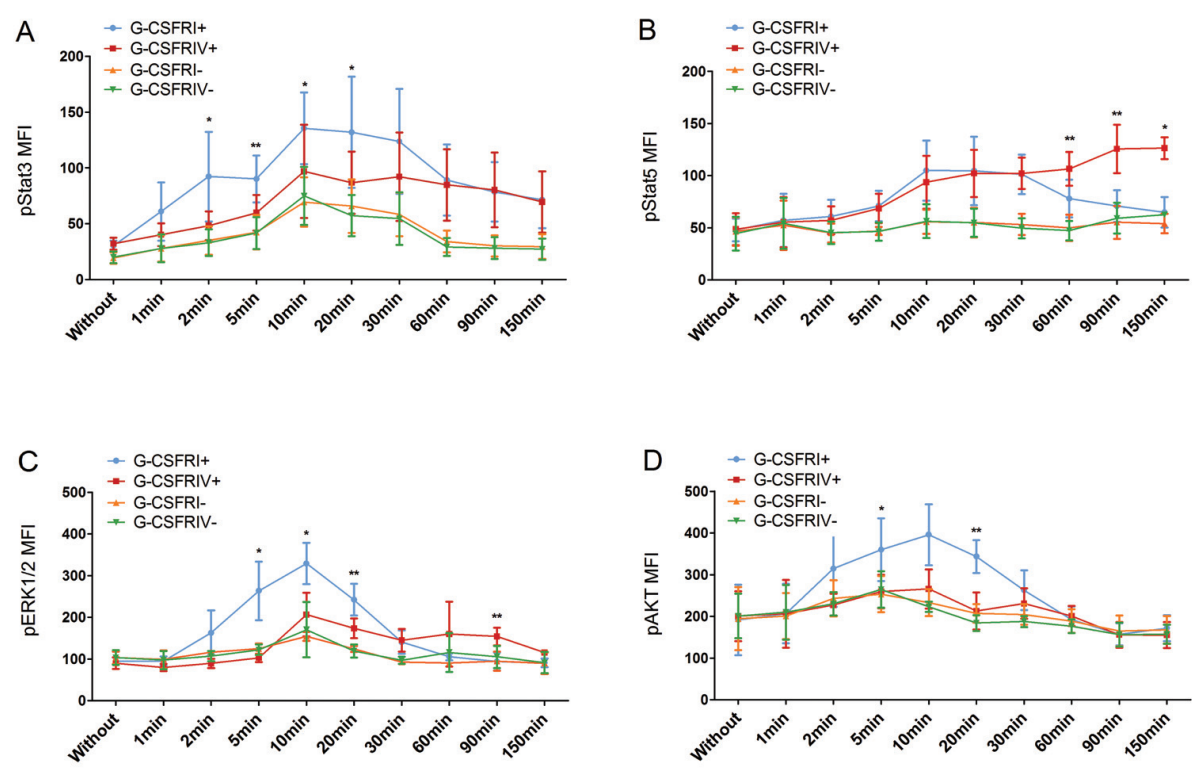

Figure 4. G-CSFRIV mediates aberrant activation of the Stat3, Stat5, ERK1/2 and AKT signaling pathways. CD34 $4^{+}$HSPCs were transduced with a lentiviral vector encoding for either G-CSFRI or G-CSFRIV sequences. Unsorted serum-starved cells $\left(1 \times 10^{5}\right)$ were stimulated with $100 \mathrm{ng} / \mathrm{mL}$ G-CSF for sequential time points from 0 to $150 \mathrm{~min}$ and signaling pathway analysis was performed. GFP expression was used to distinguish G-CSFR overexpressing and nontransduced cells. Levels of phosphorylated proteins at different time points were compared with the respective phosphorylated protein level detected without stimulation. Graph displays MFI of G-CSF-induced phosphorylated Stał3 (A), Stat5 (B), ERK1/2 (C) and AKT (D) in G-CSFRI ${ }^{+}, G_{-C S F R I V}+$, G-CSFRI ${ }^{-}$and G-CSFRIV ${ }^{-}$HSPCs. Data shown are mean \pm SD of six independent experiments. Statistically significant differences were calculated using a two-tailed Student $t$ test and are shown with asterisks ${ }^{*} p<0.05$ and $\left.{ }^{* *} P<0.01\right)$. G-CSFRI', G-CSFRI nontransduced; G-CSFRIV', G-CSFRIV nontransduced.

a Stat5 inhibitor in both the G-CSFRI ${ }^{+}$ and G-CSFRIV ${ }^{+}$HSPCs (CPD eFluor MFI: $6,456 \pm 2,351 \mathrm{G}-\mathrm{CSFRI}^{+}$with DMSO

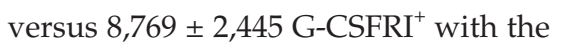
Stat5 inhibitor, $p<0.0001$, and 4,336 \pm 2,457 G-CSFRIV ${ }^{+}$with DMSO versus $6,151 \pm 2,559$ G-CSFRIV $^{+}$with the Stat5 inhibitor, $p=0.0157)$. Importantly, in the presence of the Stat 5 inhibitor, the proliferation rate of the G-CSFRIV ${ }^{+}$HSPCs decreased to a level comparable to that demonstrated by the G-CSFRI ${ }^{+}$HPSCs in the absence of the Stat5 inhibitor.

Furthermore, we investigated whether the G-CSFRIV-mediated hyperproliferative response depends on the upregulation of miR-155. We observed reduced proliferation rates in the G-CSFRIV ${ }^{+}$ HSPCs that expressed shmiR-155 compared with the G-CSFRIV ${ }^{+}$HSPCs control that expressed an empty negative control shRNA (shNC) (CPD eFluor MFI:
$5,915 \pm 1,899$ shmiR-155 versus $4,481 \pm$ 1,571 shNC, $p=0.0397$ ) (Figure 6B).

These data indicate that the proliferative advantage conferred by G-CSFRIV depends on Stat5 and miR-155. This hyperproliferative response can be inhibited by using pharmacological drugs or RNA interference technology.

\section{G-CSF Induces G-CSFRIV and miR-155 Expression in PBMCs from AML Patients}

Elevated G-CSFRIV:G-CSFRI mRNA ratios have been detected in $>50 \%$ of AML patient samples (9). In addition, miR-155 is known to be upregulated in G-CSF-mobilized CD $34^{+}$progenitor cells (22). Therefore, we investigated whether G-CSF could further induce G-CSFRIV and miR-155 expression of PBMCs from AML patients. Strikingly, G-CSF increased the G-CSFRIV:G-CSFRI mRNA ratio in PBMCs from AML patients but not in PBMCs from healthy donors (4.265 \pm 5.903 PBMCs from AML patients versus $0.9591 \pm 0.2826 \mathrm{PBMCs}$ from healthy donors, $p=0.002$ ) (Figure 7A). In addition, the RQ values of miR-155 (Figure 7B) were increased on G-CSF stimulation in the AML patient samples compared with those from healthy donors $(1.479 \pm 0.8507$ PBMCs from AML patients versus $0.8293 \pm 0.2575$ PBMCs from healthy donors, $p=0.0394$ ).

\section{DISCUSSION}

An increasing number of reports suggest that protein variants of the G-CSFR are associated with transforming and oncogenic potential $(23,24)$. Truncated G-CSFR mutants detected in severe congenital neutropenia were shown to induce prolonged Stat 5 and reduced Stat 3 activation in response to G-CSF, leading to augmented cell proliferation and defective granulocytic differentiation and eventually contributing to the transformation of severe congenital neutropenia to AML/ myelodysplastic syndrome $(17,25)$. Structurally, both G-CSFRIV and the G-CSFR truncated mutants lack three of the four tyrosine residues and the dileucine internalization motif at the C-terminal region of the cytoplasmic domain. In addition, AML and myelodysplastic syndrome patients were shown to have increased G-CSFRIV:G-CSFRI mRNA ratios $(8,9)$. Therefore, there is an unmet need to clarify the potential oncogenic role of G-CSFRIV in human progenitor cells. Several studies have consistently demonstrated that G-CSFRIV is associated with defective myeloid differentiation $(9,18)$. However, the effect of G-CSFRIV on cell proliferation remains controversial $(10,18)$. In the present study, we demonstrated with different proliferation assays that G-CSFRIV conferred a growth advantage to human HSPCs. Stat3 activation at Y704 and Y744 of G-CSFR plays a critical role in G-CSF-induced differentiation. Y704 and Y744 are absent in the G-CSFRIV protein variant. As expected, we observed a permanently reduced Stat 3 activation and delayed myeloid maturation of the 

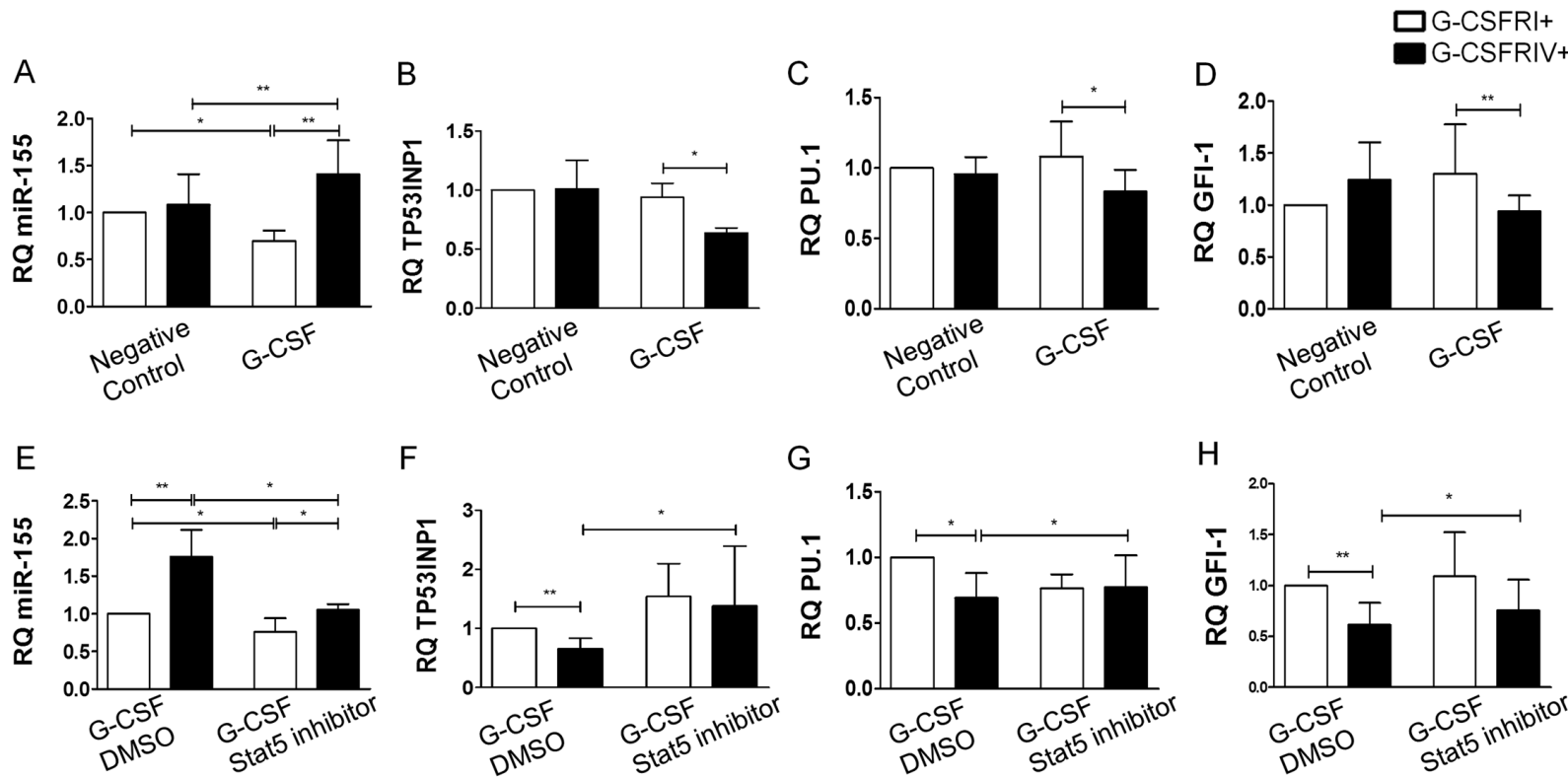

$\mathrm{F}$

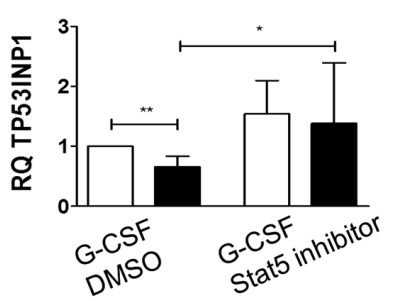

G

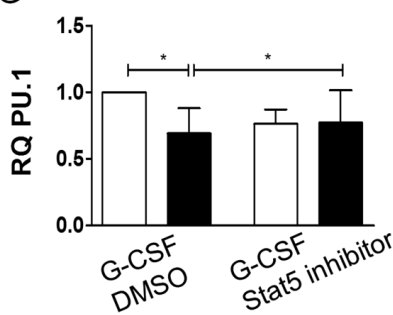

$\mathrm{H}$

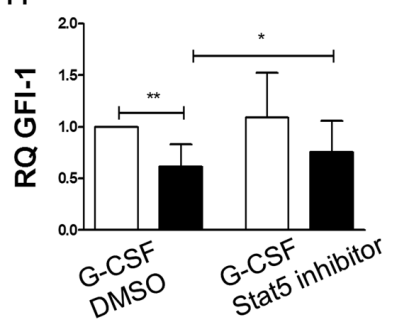

Figure 5. Sustained Stat5 activation in G-CSFRIV ${ }^{+}$SSPCs upregulates miR-155 and downregulates its target genes expression. Enriched G-CSFRI ${ }^{+}$or G-CSFRIV ${ }^{+}$HSPCs $\left(2 \times 10^{5}\right)$ were grown in presence or absence of $100 \mathrm{ng} / \mathrm{mL} \mathrm{G-CSF}$ for $1 \mathrm{~d}$. Levels of miR-155 and its candidate target genes expression were analyzed by real-time PCR. U6 or GAPDH levels were used as endogenous control of miR-155 or mRNA levels, respectively. Levels of the miR 155 and all analyzed target genes were normalized to the respective levels detected in the G-CSFRI ${ }^{+}$cells without cytokine stimulation. Graphs display RQ values of miR-155 (A), TP53INPI (B), PU.1 (C) and GFI-1 (D). G-CSFRI ${ }^{+}$or G-CSFRIV ${ }^{+}$cells $\left(2 \times 10^{5}\right)$ were stimulated with $100 \mathrm{ng} / \mathrm{mL}$ G-CSF in combination with DMSO as control or with a Stat5 inhibitor (100 $\mu \mathrm{mol} / \mathrm{L}$ ) for $1 \mathrm{~d}$. Levels of the miR 155 and all analyzed target genes were normalized to the respective levels detected in the G-CSFR $I^{+}$ cells with G-CSF plus DMSO stimulation. Graphs display RQ values of miR-155 (E), TP53INP1 (F), PU.1 (G) and GFI-1 (H). Data were shown as mean \pm SD of six independent experiments. Statistically significant differences were calculated using a two-tailed Student $t$ test and are shown with asterisks $\left({ }^{*} p<0.05\right.$ and $\left.{ }^{* *} p<0.01\right)$.

G-CSFRIV ${ }^{+}$cells compared with the G-CSFRI ${ }^{+}$cells. The docking sites and the function of G-CSF-induced AKT and ERK1/2 activation have not yet been clearly explained (26-28). Our data showed that G-CSFRIV could not mediate AKT activation upon G-CSF stimulation, and the activation of ERK1/2 remained low but sustained. These results may indicate that G-CSF-induced activation of AKT requires the terminal tyrosine residues. In addition, it is likely that ERK1/2 activation is mediated by the terminal tyrosine residues at early time

Table 1. G-CSFRIV promotes CCL2 secretion in a Stat5-dependent manner.

\begin{tabular}{lccccc} 
& \multicolumn{2}{c}{ G-CSF + DMSO } & & \multicolumn{2}{c}{ G-CSF + Stat5 inhibitor } \\
\cline { 2 - 3 } CCL2 $(\mathrm{pg} / \mathrm{mL})$ & G-CSFRI & G-CSFRIV & & G-CSFRI & G-CSFRIV \\
\hline Day 1 & $169.0 \pm 138.8$ & $637.9 \pm 381.8^{\mathrm{a}}$ & & $171.9 \pm 207.0$ & $230.3 \pm 240.3^{\mathrm{b}}$ \\
Day 3 & $128.3 \pm 105.1$ & $2413.0 \pm 2031.0^{a}$ & & $117.9 \pm 115.3$ & $492.5 \pm 301.7^{\mathrm{b}}$ \\
\hline
\end{tabular}

HSPCs were transduced with a lentiviral vector encoding either G-CSFRI or G-CSFRIV sequences. Cells were stimulated with $100 \mathrm{ng} / \mathrm{mL}$ G-CSF and in the presence of DMSO as a control or a Stat5 inhibitor $(100 \mathrm{nmol} / \mathrm{L})$ for 1 and $3 \mathrm{~d}$. Data are mean \pm SD of CCL2 levels $(\mathrm{pg} / \mathrm{mL})$ from five donors. Statistically significant differences were calculated using a Student two-tailed t test. ${ }^{a} p<0.05, \mathrm{CCL} 2$ levels detected in HSPCs containing G-CSFRI ${ }^{+}$ cells versus HSPCs containing G-CSFRIV ${ }^{+}$cells in presence of G-CSF + DMSO. ${ }^{b} p<0.05$, CCL2 levels detected in HSPCs containing G-CSFRIV ${ }^{+}$cells stimulated with G-CSF + DMSO versus stimulated with G-CSF + Stat5 inhibitor.

points and is later mediated by the membrane proximal domain. However, further studies are required to investigate the residues involved in and the clinical relevance of the G-CSFRIV-mediated aberrant $\mathrm{AKT}$ and ERK1/2 activation.

Activation of Stat 5 at the membraneproximal region has been linked to hematopoietic cell proliferation and survival (29). Inactivating the Stat5 signal is associated with receptor internalization and SOCS3 suppression $(19,30)$. In our study, we observed a prolonged Stat5 activation in the G-CSFRIV ${ }^{+}$HSPCs. This effect might be caused by the lack of the dileucine internalization motif and the recruitment site for SOCS3. In contrast to our data, Mehta et al. (8) recently showed that G-CSFRIV expression is associated with reduced G-CSF-induced Stat5 activation. However, those observations were obtained in the Ba/F3 cell line, not in primary HSPCs. Constitutive activation of 

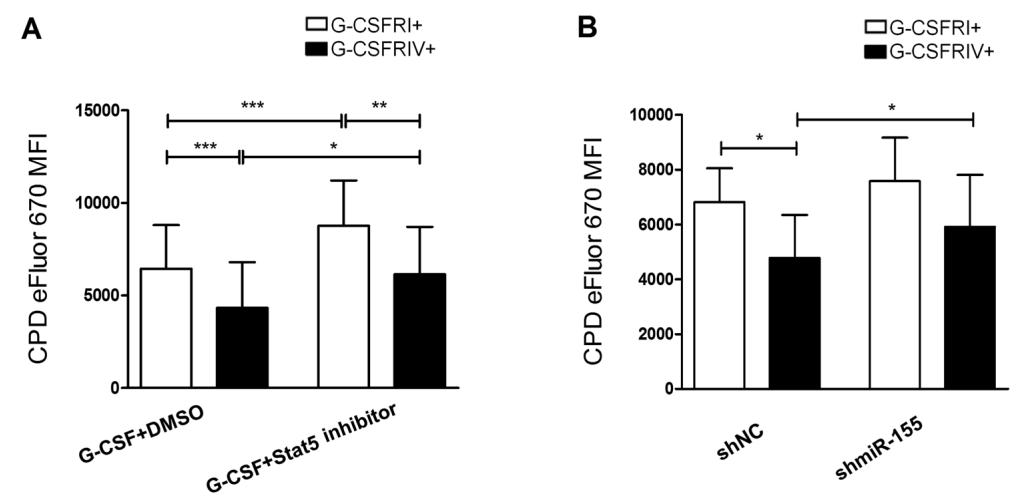

Figure 6. G-CSFRIV-mediated hyperproliferative response is compromised in the presence of a Stat5 inhibitor or shmiR-155. (A) CD34 ${ }^{+}$HSPCs were transduced with a lentiviral vector containing either G-CSFRI or G-CSFRIV encoding sequences. Cells $\left(1 \times 10^{5}\right)$ were labeled with CPD eFluor 670 and stimulated with $100 \mathrm{ng} / \mathrm{mL}$ G-CSF together with DMSO as control or with a Stat5 inhibitor ( $100 \mu \mathrm{mol} / \mathrm{L}$ ) for $3 \mathrm{~d}$ followed by FACS analysis. GFP expression was used to distinguish G-CSFR overexpressing and nontransduced cells. Graph displays MFI of CPD eFluor 670 on G-CSFRI ${ }^{+}$and G-CSFRIV ${ }^{+}$HSPCs. Data are mean \pm SD of eight independent experiments. (B) CD34+ HSPCs were transduced with a G-CSFRI or G-CSFRIV encoding lentiviral vector together with shmiR-155 or shNC. GFP and DsRed were used to distinguish transduced and nontransduced cells. Cells $\left(1 \times 10^{5}\right)$ were labeled with CPD efluor 670 and stimulated with $100 \mathrm{ng} / \mathrm{mL}$ G-CSF for $3 \mathrm{~d}$ followed by FACS analysis. Graph displays MFI of CPD efluor 670 on G-CSFRI ${ }^{+}$shNC, G-CSFRI ${ }^{+}$shmiR-155, G-CSFRIV ${ }^{+}$shNC and G-CSFRIV ${ }^{+}$shmiR-155 cells. The data shown are mean \pm SD of four independent experiments. Statistically significant differences were calculated using a two-tailed Student $t$ test and are shown with asterisks $\left({ }^{*} p<0.05,{ }^{* *} p<0.01\right.$ and $\left.{ }^{* * *} p<0.001\right)$.

\section{A}

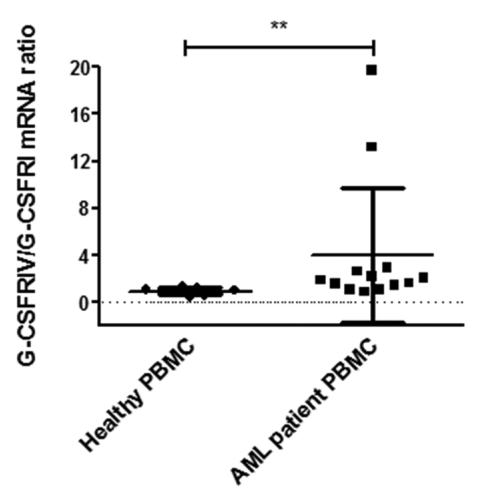

B

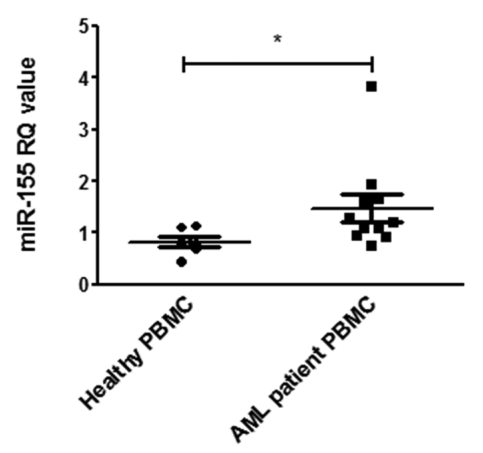

Figure 7. G-CSF induces G-CSFRIV and miR-155 expression in PBMCs from AML patients. (A) PBMCs collected from 13 AML patients and 8 healthy donors were cultured with or without $100 \mathrm{ng} / \mathrm{mL}$ G-CSF for $2 \mathrm{~d}$ and subjected to total RNA extraction and G-CSFRI and G-CSFRIV mRNA quantification. GAPDH levels were used as endogenous control. G-CSFRIV:G-CSFRI ratio was normalized to the respective ratio detected in the cells cultured without G-CSF. Graph displays mean \pm SD of G-CSFRIV:G-CSFRI mRNA ratios. (B) PBMCs collected from 10 AML patients and 6 healthy donors were cultured with or without G-CSF for $2 \mathrm{~d}$ and subjected to total RNA extraction and miR-155 quantification. U6 levels were used as endogenous control. The level of miR 155 was normalized to the respective level detected in the cells cultured without G-CSF. Graph displays RQ values (mean $\pm S D$ ) of miR-155. Statistically significant differences were calculated using a twotailed Student $t$ test and are shown with asterisks $\left({ }^{*} p<0.05\right.$ and $\left.{ }^{* *} p<0.01\right)$.
Stat 5 has been observed in many hematological and solid malignancies, leading to the deregulation of the Stat 5 target genes and consequently malignant transformation (31). Although several Stat5 targets are known, identifying novel Stat5 targets is still required to decipher the detailed transformation process.

Emerging evidence shows that specific miRNAs are involved in hematopoiesis. miR-155 is highly expressed in hematopoietic cells and has been linked to a variety of solid tumors as well as lymphoma and leukemia (32). The forced expression of miR-155 was demonstrated to trigger a myelo-proliferative disorder that exhibits many preleukemic aspects (33). Clinically, miR-155 was found to be overexpressed in highly proliferative AML subtypes (French-American-British [FAB] subtypes M4 and M5, as well as in patients with FLT3 internal tandem duplication [FLT3-ITD]) $(21,34)$ and was identified as an unfavorable prognostic factor for AML patients with a normal karyotype (35). miR-155 targets genes that are associated with cell viability, apoptosis and tumor suppression. Among these genes, PU.1 (36) and GFI-1 are crucial for myeloid differentiation and tumor suppression. TP53INP1 has antiproliferative and proapoptotic activity (37). Recently, the study by Kopp et al. (38) showed that the miR-155 host gene B-cell integration cluster (BIC) was a transcriptional target of Stat5, and the Stat5/BIC/miR-155 pathway was associated with the proliferation of malignant T cells. Notably, in our study, the prolonged activation of Stat5, correlated with increased levels of miR-155 and reduced levels of miR-155 target genes (PU.1, GFI-1 and TP53INP1), could be observed in the G-CSFRIV ${ }^{+}$HSPCs on G-CSF stimulation. To confirm the interaction between Stat 5 and miR-155, we analyzed miR-155 expression after Stat5 inhibition. As expected, in the presence of the Stat5 inhibitor, G-CSF-induced upregulation of miR-155 in the G-CSFRIV ${ }^{+}$ HSPCs was significantly abrogated. Accordingly, the expression of the miR-155 target genes was unchanged. Our data 


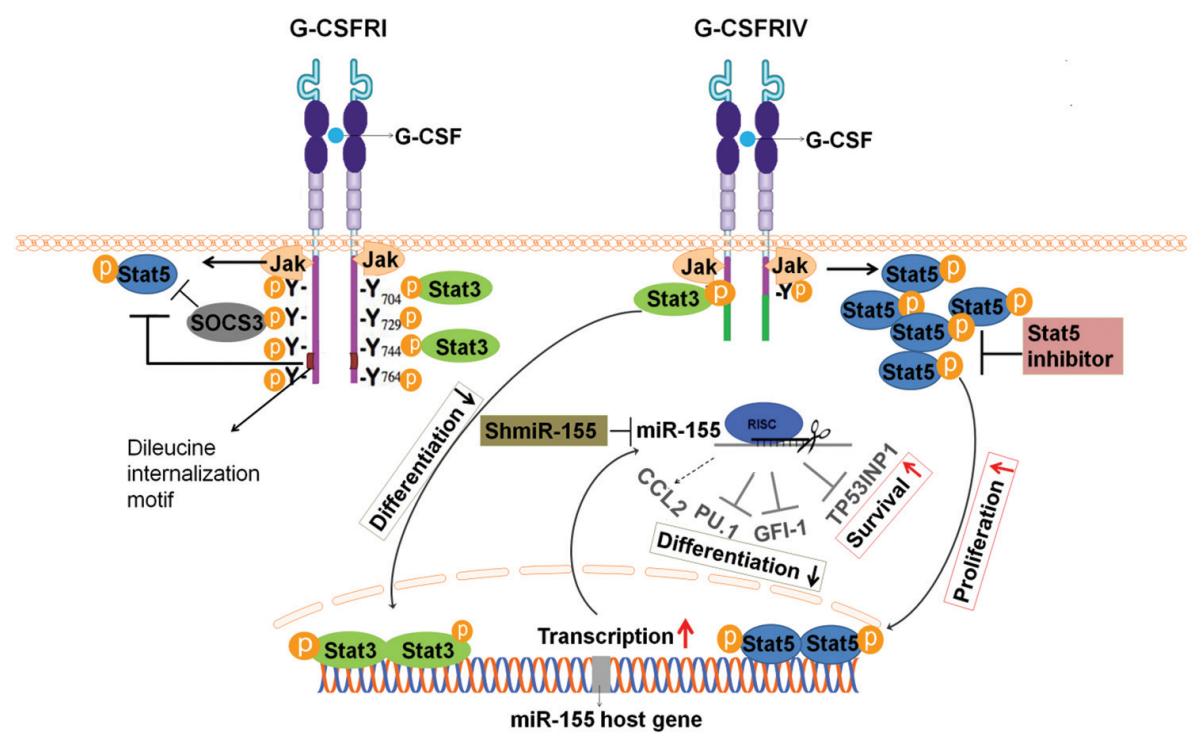

Figure 8. Schema of structure and signaling pathway of G-CSFRI and G-CSFRIV. G-CSFRI consists of an extracellular domain, a transmembrane domain and a cytoplasmic domain. The membrane-proximal region of the cytoplasmic domain has been linked to activation of Jak and Stat5. The C-terminal end of G-CSFR cytoplasmic domain contains the dileucine internalization motif and four conserved tyrosine residues. Y704 and Y744 recruit Stat3, and Y729 is a docking site for SOCS3. The dileucine internalization motif and SOCS3 could inhibit Stat5 activation. In the alternatively spliced G-CSFRIV, 87 amino acids at the C-terminal end of the cytoplasmic domain including Y729, Y744, Y764 and dileucine internalization motif are replaced by 34 amino acids of a novel sequence. In response to G-CSF, the G-CSFR forms homodimers and leads to rapid Jak phosphorylation and subsequently the activation of Stat3, Stat5 and SOCS3. The Stat proteins then dimerize, translocate to the nucleus and bind to specific DNA recognition sites and modulate the expression of the target genes. G-CSFRIV induces prolonged Stat5 activation, which causes an upregulation of the miR-155 host gene. miR-155 further downregulates the levels of its target genes including PU. 1, GFI-1 and TP53INP1, contributing to growth and survival advantage, increased CCL2 secretion as well as defective differentiation of hematopoietic stem and progenitor cells. The Stat5 inhibitor and shmiR-155 may abrogate G-CSFRIV-mediated Stat5/miR-155/miR-155-targets pathway.

supported the previous study of Kopp et al. indicating that miR-155 is a transcriptional target of Stat5.

CCL2 is a strong chemotactic factor for monocytes and macrophages, which are the main source of endogenous G-CSF (39). In many malignant diseases, a causal role has recently been attributed to inflammatory factors, including CCL2, in the development and progression of cancer (40). It is interesting to note that CCL2 deficiency was shown to impair the secretion of G-CSF (41). In addition, miR-155 was recently found to indirectly regulate CCL2 expression (42). In this study, we observed that the G-CSFRIV ${ }^{+}$ HSPCs secreted markedly higher levels of CCL2 compared with the G-CSFRI ${ }^{+}$ HSPCs. Furthermore, the CCL2 levels were correlated with Stat 5 activation in the G-CSFRIV ${ }^{+}$HSPCs. These observations may indicate that CCL2 is a downstream target of Stat5 or miR-155, and CCL2 might contribute to the oncogenic potential of G-CSFRIV by recruiting G-CSF-secreting monocytes and macrophages as well as favoring cell metastasis. However, further studies are needed to prove this concept and to elucidate the detailed mechanism.

Importantly, we further showed that G-CSF could induce G-CSFRIV and miR155 expression in the PBMCs from AML patients. Hence, the use of G-CSF to treat
AML patients, especially those carrying a high G-CSFRIV:G-CSFRI ratio, might represent a major concern. Our data indicate that individuals carrying an elevated G-CSFRIV:G-CSFRI ratio are more susceptible to this undesirable effect of G-CSF, which leads to the upregulation of aberrant levels of miR-155, thus increasing the risk of de novo leukemogenicity or relapse.

\section{CONCLUSION}

Overall, the present study shows that G-CSF induces sustained Stat5 activation, leading to the upregulation of miR155 and the consequent inhibition of the miR-155-targeted genes PU.1, GFI-1 and TP53NP1. Moreover, the Stat5/miR-155/ miR-155 targets pathway promotes HSPC proliferation, survival and secretion of CCL2 and delays the myeloid differentiation of HSPCs, which indicate leukemic transformation. Importantly, the inhibition of Stat 5 or miR-155 significantly decreases the G-CSFRIV-mediated hyperproliferation (Figure 8). Our data provide direct evidence for the leukomogenic role of G-CSFRIV in human primary CD $34^{+}$HSPCs. To our knowledge, we describe for the first time that the leukemogenic potential of G-CSFRIV is associated with the Stat5-dependent dysregulation of miR-155 and the target genes of this miRNA. Considering the broad applications of G-CSF in the clinic, it is highly recommended that the G-CSFRIV expression levels of the progenitor cells from patients and stem cell donors be determined before G-CSF administration. And those patients or healthy HSPCs donors harboring the elevated G-CSFRIV:G-CSFRI mRNA ratio may require alternative treatments to G-CSF or avoid high G-CSF doses. Moreover, our data suggest that Stat 5 or miR155 inhibitors strongly contribute to the abrogation of the leukemogenic potential of the G-CSFRIV ${ }^{+}$HSPCs. This result may represent a novel therapeutic approach to decreasing the risk of developing leukemia or a disease relapse in patients with an elevated G-CSFRIV:G-CSFRI ratio. 


\section{ACKNOWLEDGMENTS}

This work was supported by the Excellence Cluster Rebirth (EXC Unit 6.3). The authors acknowledge Stefanie Vahlsing for her excellent technical assistance. In addition, the authors are grateful to Matthias Ballmaier for help and guidance in the FACS sorting facility.

\section{DISCLOSURE}

The authors declare that they have no competing interests as defined by Molecular Medicine, or other interests that might be perceived to influence the results and discussion reported in this paper.

\section{REFERENCES}

1. Demetri GD, Griffin JD. (1991) Granulocyte colony-stimulating factor and its receptor. Blood. 78:2791-808.

2. Touw IP, van de Geijn GJ. (2007) Granulocyte colony-stimulating factor and its receptor in normal myeloid cell development, leukemia and related blood cell disorders. Front. Biosci. 12:800-15.

3. Beekman R, Touw IP. (2010) G-CSF and its receptor in myeloid malignancy. Blood. 115:5131-6.

4. Yoshikawa A, Murakami H, Nagata S. (1995) Distinct signal transduction through the tyrosinecontaining domains of the granulocyte colonystimulating factor receptor. EMBO J. 14:5288-96.

5. Richards MK, Liu F, Iwasaki H, Akashi K, Link DC. (2003) Pivotal role of granulocyte colony-stimulating factor in the development of progenitors in the common myeloid pathway. Blood. 102:3562-8.

6. Hermans MH, et al. (2003) Signaling mechanisms coupled to tyrosines in the granulocyte colonystimulating factor receptor orchestrate G-CSFinduced expansion of myeloid progenitor cells. Blood. 101:2584-90.

7. Bernard T, Gale RE, Linch DC. (1996) Analysis of granulocyte colony stimulating factor receptor isoforms, polymorphisms and mutations in normal haemopoietic cells and acute myeloid leukaemia blasts. Br. J. Haematol. 93:527-33.

8. Mehta HM, et al. (2010) Alternatively spliced, truncated GCSF receptor promotes leukemogenic properties and sensitivity to JAK inhibition. Leukemia. 28:1041-51.

9. White SM, Ball ED, Ehmann WC, Rao AS, Tweardy DJ. (1998) Increased expression of the differentiation-defective granulocyte colony-stimulating factor receptor mRNA isoform in acute myelogenous leukemia. Leukemia. 12:899-906.

10. Sloand EM, et al. (2006) Granulocyte colonystimulating factor preferentially stimulates proliferation of monosomy 7 cells bearing the isoform IV receptor. Proc. Natl. Acad. Sci. U. S. A. 103:14483-8.

11. Ehlers S, et al. (2010) Granulocyte colony-stimulating factor (G-CSF) treatment of childhood acute myeloid leukemias that overexpress the differentiation-defective G-CSF receptor isoform
IV is associated with a higher incidence of relapse. J. Clin. Oncol. 28:2591-7.

12. Kluiver J, Kroesen BJ, Poppema S, van den Berg A. (2006) The role of microRNAs in normal hematopoiesis and hematopoietic malignancies. Leukemia. 20:1931-6.

13. Elton TS, Selemon H, Elton SM, Parinandi NL. (2013) Regulation of the MIR155 host gene in physiological and pathological processes. Gene. 532:1-12.

14. Swerdlow SH, et al. (eds.). (2008) WHO Classification of Tumours of Haematopoietic and Lymphoid Tissues. 4th ed. Lyon (France): International Agency for Research on Cancer. 439 pp.

15. Chen C, et al. (2005) Real-time quantification of microRNAs by stem-loop RT-PCR. Nucleic Acids Res. 33:e179.

16. De Waele M, et al. (2001) Growth factor receptor profile of CD34+ cells in AML and B-lineage ALL and in their normal bone marrow counterparts. Eur. J. Haematol. 66:178-87.

17. Hunter MG, Avalos BR. (2000) Granulocyte colonystimulating factor receptor mutations in severe congenital neutropenia transforming to acute myelogenous leukemia confer resistance to apoptosis and enhance cell survival. Blood. 95:2132-7.

18. White SM, Alarcon MH, Tweardy DJ. (2000) Inhibition of granulocyte colony-stimulating factormediated myeloid maturation by low level expression of the differentiation-defective class IV granulocyte colony-stimulating factor receptor isoform. Blood. 95:3335-40.

19. van de Geijn GJ, Gits J, Aarts LH, HeijmansAntonissen C, Touw IP. (2004) G-CSF receptor truncations found in SCN/AML relieve SOCS3controlled inhibition of STAT5 but leave suppression of STAT3 intact. Blood. 104:667-74

20. Nguyen-Jackson HT, Zhang H,Watowich SS. (2012) G-CSF Receptor Structure, Function, and Intracellular Signal Transduction. In: Twenty Years of G-CSF: Clinical and Nonclinical Discoveries. Molineux G, Arvedson T, Foote MA (eds.) Springer Basel, Basel, pp. 83-105.

21. Marcucci G, et al. (2008) MicroRNA Expression in Cytogenetically Normal Acute Myeloid Leukemia. N. Engl. J. Med. 358:1919-28.

22. Donahue RE, et al. (2009) Plerixafor (AMD3100) and granulocyte colony-stimulating factor (G-CSF) mobilize different CD34+ cell populations based on global gene and microRNA expression signatures. Blood. 114:2530-41.

23. Germeshausen M, et al. (2005) An acquired G-CSF receptor mutation results in increased proliferation of CMML cells from a patient with severe congenital neutropenia. Leukemia. 19:611-7.

24. Touw IP, Palande K, Beekman R. (2013) Granulocyte colony-stimulating factor receptor signaling: implications for G-CSF responses and leukemic progression in severe congenital neutropenia. Hematol. Oncol. Clin. North Am. 27:61-73, viii.

25. Ward AC, et al. (2008) Functional interaction between mutations in the granulocyte colony-stimulating factor receptor in severe congenital neutropenia. Br. J. Haematol. 142:653-6.

26. Zhu QS, Robinson LJ, Roginskaya V, Corey SJ.
(2004) G-CSF-induced tyrosine phosphorylation of Gab2 is Lyn kinase dependent and associated with enhanced Akt and differentiative, not proliferative, responses. Blood. 103:3305-12.

27. Cassinat B, et al. (2011) New role for granulocyte colony-stimulating factor-induced extracellular signal-regulated kinase $1 / 2$ in histone modification and retinoic acid receptor alpha recruitment to gene promoters: relevance to acute promyelocytic leukemia cell differentiation. Mol. Cell. Biol. 31:1409-18.

28. Kamezaki K, et al. (2005) Roles of Stat3 and ERK in G-CSF signaling. Stem Cells. 23:252-63.

29. Dong F, et al. (1998) Stimulation of Stat5 by granulocyte colony-stimulating factor (G-CSF) is modulated by two distinct cytoplasmic regions of the G-CSF receptor. J. Immunol. 161:6503-9.

30. Hunter MG, et al. (2008) Divergent pathways in COS-7 cells mediate defective internalization and intracellular routing of truncated G-CSFR forms in SCN/AML. PLoS One. 3:e2452.

31. Ferbeyre G, Moriggl R. (2011) The role of Stat5 transcription factors as tumor suppressors or oncogenes. Biochim. Biophys. Acta. 1815:104-14.

32. Costinean S, et al. (2006) Pre-B cell proliferation and lymphoblastic leukemia/high-grade lymphoma in E(mu)-miR155 transgenic mice. Proc. Natl. Acad. Sci. U. S. A. 103:7024-9.

33. O'Connell RM, et al. (2008) Sustained expression of microRNA-155 in hematopoietic stem cells causes a myeloproliferative disorder. J. Exp. Med. 205:585-94.

34. Zhu YD, et al. (2011) Distinctive microRNA signature is associated with the diagnosis and prognosis of acute leukemia. Med. Oncol. 29:2323-31.

35. Faraoni I, et al. (2012) miR-424 and miR-155 deregulated expression in cytogenetically normal acute myeloid leukaemia: correlation with NPM1 and FLT3 mutation status. J. Hematol. Oncol. 5:26.

36. Thompson RC, Herscovitch M, Zhao I, Ford TJ, Gilmore TD. (2011) NF-kappaB down-regulates expression of the B-lymphoma marker CD10 through a miR-155/PU.1 pathway. J. Biol. Chem. 286:1675-82.

37. Wei Q, Li YX, Liu M, Li X, Tang H. (2012) miR$17-5 p$ targets TP53INP1 and regulates cell proliferation and apoptosis of cervical cancer cells. IUBMB Life. 64:697-704.

38. Kopp KL, et al. (2013) STAT5-mediated expression of oncogenic miR-155 in cutaneous T-cell lymphoma. Cell Cycle. 12:1939-47.

39. Avalos BR. (1996) Molecular analysis of the granulocyte colony-stimulating factor receptor. Blood. 88:761-77.

40. Conti I, Rollins BJ. (2004) CCL2 (monocyte chemoattractant protein-1) and cancer. Semin. Cancer Biol. 14:149-54.

41. Strecker J-K, et al. (2011) Monocyte chemoattractant protein-1-deficiency impairs the expression of IL-6, IL-1 $\beta$ and G-CSF after transient focal ischemia in mice. PLoS One. 6:e25863.

42. Nazari-Jahantigh M, et al. (2012) MicroRNA-155 promotes atherosclerosis by repressing $\mathrm{Bcl} 6$ in macrophages. J. Clin. Invest. 122:4190-202. 